\title{
DEVELOPMENT OF A MULTIPLEX PCR FOR SIMULTANEOUS DETECTION OF PASTEURELLA MULTOCIDA, MANNHEIMIA HAEMOLYTICA AND TRUEPERELLA PYOGENES
}

\author{
Wenlong ZHANG ${ }^{\dagger, 1,2,3}$, Xiaodan LIU ${ }^{1}$, Mengcheng $\mathrm{LiU}^{1}$, Bo MA ${ }^{1,2}$, Li Xu \\ and Junwei WANG ${ }^{\ddagger 1,2}$ \\ ${ }^{1}$ Department of Preventive Veterinary Medicine, College of Veterinary Medicine, \\ Northeast Agricultural University, Harbin, Heilongjiang 150030, P. R. China; \\ ${ }^{2}$ Northeastern Science Inspection Station, China Ministry of Agriculture Key Laboratory \\ of Animal Pathogen Biology, P. R. China; ${ }^{3}$ College of Animal Science and Technology, \\ Northeast Agricultural University, Harbin, Heilongjiang 150030, P. R. China
}

(Received 13 December 2016; accepted 12 July 2017)

Pasteurella multocida, Mannheimia haemolytica and Trueperella pyogenes are three bacterial pathogens closely associated with the bovine respiratory disease complex (BRDC). In the current study, a multiplex PCR for the simultaneous detection of these three bacteria in cultures was established. After serial optimisation, the detection limit of the method for the genomic DNA of the three bacteria was $40 \mathrm{pg} / \mu \mathrm{l}$. The method could detect the genomic DNA of these three bacteria but not the genomic DNA of seven other bacterial strains. Together with the bacterial enrichment technology, the multiplex PCR could be used for detecting the three bacteria in animal tissues. This method might be valuable for speeding up laboratory diagnosis and directing the treatment of BRDC to these three bacterial pathogens.

Key words: Multiplex PCR, Pasteurella multocida, Mannheimia haemolytica, Trueperella pyogenes

The bovine respiratory disease complex (BRDC) is an economically significant cattle disease (Grissett et al., 2015), the pathogenesis of which exhibits complexity and involves many different infectious agents (Confer, 2009; Griffin, 2010). A respiratory environment favourable for the colonisation and replication of several pathogenic bacteria is established when stressors, such as weaning and shipping, or infections caused by several primary respiratory pathogens (such as bovine herpesvirus type 1 , bovine parainfluenza virus type 3 , bovine respiratory syncytial virus and bovine viral diarrhoea virus) compromise the immune defence of animals (Confer, 2009; Kishimoto et al., 2017). In such cases, bacteria invade the lower respiratory tract of cattle and induce severe bacterial pneumonia

${ }^{\dagger}$ Corresponding author; E-mail: zhangwenlong@neau.edu.cn; Phone: 0086 (451) 55190385

†Corresponding author; E-mail: jwwang@neau.edu.cn; Phone/Fax: 0086 (451) 55190727 
which is the most fatal form of bovine respiratory disease (BRD) (Confer, 2009; Griffin, 2010). Complex infections attributed to various pathogens are usually necessary for the manifestation of signs of BRDC (Kishimoto et al., 2017). For this reason, the causative pathogens of this disease cannot be determined easily.

Pasteurella multocida, Mannheimia haemolytica and Trueperella pyogenes are three bacterial pathogens that can often be isolated from cattle with BRDC (Angen et al., 2009). These bacteria are ubiquitous and normal nasopharyngeal commensals of healthy bovines, and they become pathogenic when the immune system of their host is overwhelmed by adverse factors (Confer, 2009; Griffin, 2010). Thus, these disease-causing organisms should be precisely and rapidly identified, and suitable measures should be timely applied to prevent and control the disease.

Several traditional methods for pathogen detection are based on a one assay-one pathogen approach, and some of them, such as pathogen isolation, are often time consuming (Kardos et al., 2007; Hu et al., 2011). As such, traditional methods are insufficient for the rapid diagnosis of BRDC. Conversely, timesaving methods that can detect several pathogens in a single run are possibly appropriate for rapidly determining the aetiology of BRDC. For example, multiplex polymerase chain reaction (PCR) assays with well-designed primer pairs can efficiently and specifically amplify the nucleic acid of microorganisms. Therefore, this method has been extensively used to identify microorganisms (Elbrissi et al., 2017; Moriconi et al., 2017). With regard to BRDC diagnosis, some real-time multiplex PCR methods have been established for the detection of BRDC-related viral pathogens (Horwood and Mahony, 2011; Thonur et al., 2012). However, effective and efficient multiplex PCR methods targeting BRDC-related opportunistic bacterial pathogens are still eagerly needed.

In the current study, we established a multiplex PCR for the simultaneous detection of P. multocida, M. haemolytica and T. pyogenes in cultures. The multiplex PCR, together with the bacterial enrichment technology, could be used to detect these three bacteria from animal tissues. This method might be valuable for speeding up laboratory diagnosis and directing the treatment of BRDC.

\section{Material and methods}

\section{Bacterial strains and growth conditions}

The bacterial strains used in the current study are listed in Table 1. Pasteurella multocida was cultured at $37^{\circ} \mathrm{C}$ in Martin broth medium (Beijing AoBoXing Bio-Tech Co., Ltd), while M. haemolytica and T. pyogenes were cultured at $37{ }^{\circ} \mathrm{C}$ in Martin broth with $5 \%$ fetal bovine serum. Other bacterial strains were cultured in appropriate media under the recommended culture conditions. 
Table 1

Bacteria used in the current study and the source of the strains

\begin{tabular}{ll}
\hline Bacterial strains & \multicolumn{1}{c}{ Source } \\
\hline Pasteurella multocida & CVCC447 (bovine) \\
Mannheimia haemolytica & CVCC3842 (bovine) \\
Trueperella pyogenes & Laboratory isolated (bovine) \\
Staphylococcus aureus & Laboratory isolated (bovine) \\
Streptococcus suis & Laboratory isolated (bovine) \\
Micrococcus luteus & Laboratory isolated (bovine) \\
Salmonella typhimurium & CMCC (B) 50115 (unknown) \\
Corynebacterium pseudotuberculosis & Laboratory isolated (bovine) \\
Clostridium perfringens & CVCC39 (bovine) \\
Escherichia coli & Laboratory isolated (bovine) \\
\hline
\end{tabular}

CVCC: China Veterinary Culture Collection Center; CMCC: National Center for Medical Culture Collections

\section{Target gene and primers}

The KMT1 gene of P. multocida (Townsend et al., 1998), the argininebinding periplasmic protein 2 and the leukotoxin acyl transferase (artJ-lktC) gene of M. haemolytica (Angen et al., 2009), as well as the plo gene of T. pyogenes (Billington et al., 1997), which are the previously identified conserved genes of these three organisms, were chosen as the targets of the multiplex PCR established in the current study. The nucleotide sequences of these genes as references were retrieved from the GenBank, and the primers targeting these genes (Table 2) were designed using Primer 5.0 and synthesised by BGI Tech. The theoretical specificity of the primers was verified through BLAST analysis (http://www.ncbi.nlm.nih.gov/BLAST/Blast.cgi).

Table 2

Target gene of the multiplex PCR and sequences of the primers

\begin{tabular}{llll}
\hline Bacteria & Target genes & Name and sequences of the primers (5'-3') & $\begin{array}{c}\text { Product } \\
\text { length } \\
(\mathrm{bp})\end{array}$ \\
\hline $\begin{array}{l}\text { P. multocida } \\
\text { KMT1 }\end{array}$ & $\begin{array}{l}\text { kmt. F: TATCCGCTATTTACCCAG } \\
\text { kmt. R: TGTAAACGAACTCGCCAC }\end{array}$ & 456 \\
\hline M. haemolytica & artJ-lktC & $\begin{array}{l}\text { la. F: TATAAGGATTACCACTTTAACGCA } \\
\text { la. R: ATAATCAGAAGAGAAAAAGGAGTGT }\end{array}$ & 251 \\
\hline T. pyogenes & plo & $\begin{array}{l}\text { plo. F: CAGTCAAGGGTGAGTCTATT } \\
\text { plo. R: CTTGAACTCTGTGGAAA }\end{array}$ & 773 \\
\hline
\end{tabular}




\section{DNA extraction}

The total DNA of the organisms was extracted using the Genomic DNA Preparation Mini Kit (Bioteke, China) according to the manufacturer's instructions.

\section{Establishment and optimisation of the multiplex PCR assay}

The PCR assay was performed in a $25-\mu \mathrm{L}$ total reaction volume containing $2.5 \mu \mathrm{L} 10 \times$ PCR Buffer, $100 \mathrm{ng}$ genomic DNA of each organism, and $0.25 \mu \mathrm{L}$ $r$ Taq DNA polymerase [Takara Biotechnology (Dalian) Co. Ltd]. The effect of the following parameters on the PCR reaction was evaluated: final concentration of $\mathrm{Mg}^{2+}$ from $0 \mathrm{mM}$ to $4 \mathrm{mM}$, final concentration of dNTPs from $0 \mathrm{mM}$ to $0.4 \mathrm{mM}$, final concentration of each pair of primers from $40 \mathrm{nM}$ to $200 \mathrm{nM}$, annealing temperature from $40{ }^{\circ} \mathrm{C}$ to $64{ }^{\circ} \mathrm{C}$, annealing time from $20 \mathrm{~s}$ to $50 \mathrm{~s}$, and number of amplification cycles from 20 to 40 .

\section{Determination of the specificity of the multiplex PCR}

The products of the multiplex PCR were cloned into pMD18-T vectors and then sequenced.

Pasteurella multocida, M. haemolytica, T. pyogenes and seven other bacteria (listed in Table 1) were used to confirm the specificity of the multiplex PCR. The genomic DNAs of these organisms were extracted as described above and adjusted to a concentration of $100 \mathrm{ng} / \mu \mathrm{L}$. A total of $1 \mu \mathrm{L}$ genomic DNA was then used as the template for the multiplex PCR.

\section{Determination of the sensitivity of the multiplex PCR}

To determine the detection limit of the multiplex PCR assay, serial 10-fold diluted genomic DNA of $P$. multocida, M. haemolytica and $T$. pyogenes were prepared. Subsequently, multiplex PCR assays were performed using the diluted genomic DNA as a template. Conventional PCR assays with each of the three primer pairs were performed under optimised conditions for multiplex PCR by utilising diluted genomic DNA as a template to determine if the simultaneous existence of three primer pairs in the PCR mixture leads to the reduction of sensitivity of the method in detecting any of the three organisms.

Practicability of the multiplex PCR in detecting P. multocida, M. haemolytica and $\mathrm{T}$. pyogenes in experimentally infected mice

Twenty mice were randomly divided into four groups (Groups A, B, C and D). The mice in Groups A, B and C received, respectively, an intraperitoneal inoculation of $P$. multocida, M. haemolytica or T. pyogenes culture in a dose of $0.5 \mathrm{~mL}$ per mouse. Mice in Group D received $0.5 \mathrm{~mL}$ Martin broth intraperito- 
neally and served as control. The hearts, livers, spleens, lungs, kidneys and intestinal contents of dead mice were harvested. The samples were cut into small pieces and homogenised, and then $50 \mu \mathrm{L}$ of each homogenate was inoculated into the corresponding liquid culture medium and incubated at $37{ }^{\circ} \mathrm{C}$ for $5 \mathrm{~h}$. A total of $1 \mathrm{~mL}$ of each culture was harvested and used for extracting the genomic DNA as described above. Then multiplex PCR was performed using this genomic DNA as template. Bacterial isolation and Gram staining were also performed.

Detection of the three bacteria in artificially contaminated bovine tissues

Any two or all of the three bacterial cultures of equal volume were mixed. A total of $1 \mathrm{~mL}$ of any of the three bacterial cultures and each of the mixtures were added to $1 \mathrm{~g}$ bovine tissue (lung or lymph node), which were free of the three bacteria. Then the tissues were homogenised. A total of $50 \mu \mathrm{L}$ each of the homogenates were inoculated into a liquid culture medium and then incubated at $37^{\circ} \mathrm{C}$ for $5 \mathrm{~h}$. Genomic DNA of the bacteria was extracted as described above. Then multiplex PCR was performed using these genomic DNA as template.

\section{Results}

\section{Optimisation of the reaction mixture and protocol of the multiplex PCR}

After a series of optimisation, the reaction of the $25-\mu \mathrm{L}$ mixture containing $2.5 \mu \mathrm{L} 10 \times$ PCR buffer $\left(\mathrm{Mg}^{2+}\right.$ free), $0.25 \mu \mathrm{L} r$ Taq DNA polymerase, $160 \mu \mathrm{M}$ dNTP, $1.6 \mathrm{mM} \mathrm{Mg}^{2+}, 40 \mathrm{nM}$ kmt.F/kmt.R, $100 \mathrm{nM}$ la.F/la.R, $80 \mathrm{nM}$ plo.F/plo.R and templates was determined. The amplification conditions comprised initial denaturation at $94{ }^{\circ} \mathrm{C}$ for $5 \mathrm{~min}$, followed by 40 cycles of $94{ }^{\circ} \mathrm{C}$ for $30 \mathrm{~s}, 47^{\circ} \mathrm{C}$ for $30 \mathrm{~s}, 72{ }^{\circ} \mathrm{C}$ for $50 \mathrm{~s}$, and a final extension at $72{ }^{\circ} \mathrm{C}$ for $7 \mathrm{~min}$.

\section{Determination of the specificity of the multiplex PCR}

To evaluate the specificity of the multiplex PCR, the three amplicons were first harvested and sequenced. The results showed that the nucleotide sequences of the 456, 773 and $251 \mathrm{bp}$ amplicons were highly matched with the nucleotide sequences of the corresponding reference genes from P. multocida $(98.03 \%), M$. haemolytica $(98.58 \%)$ and T. pyogenes $(100 \%)$ submitted to GenBank, respectively. This result suggests that the multiplex PCR could specifically amplify the target genes when the corresponding templates existed in the reaction mixture.

Then the specificity of the multiplex PCR was verified using seven other bacteria that were isolated from the bovine specimens. The results in Fig. 1 suggest that when any, or all, of the genomic DNA of P. multocida, M. haemolytica and $T$. pyogenes existed in the reaction mixture, the corresponding amplicon(s) 
could be observed in the agarose gel. In contrast, any genomic DNA from other microorganisms used in the reaction mixture could not be detected by the multiplex PCR in the current study.

The results suggest that the multiplex PCR could be used for specifically detecting the corresponding target genes and diagnosing diseases caused by these three bacteria.

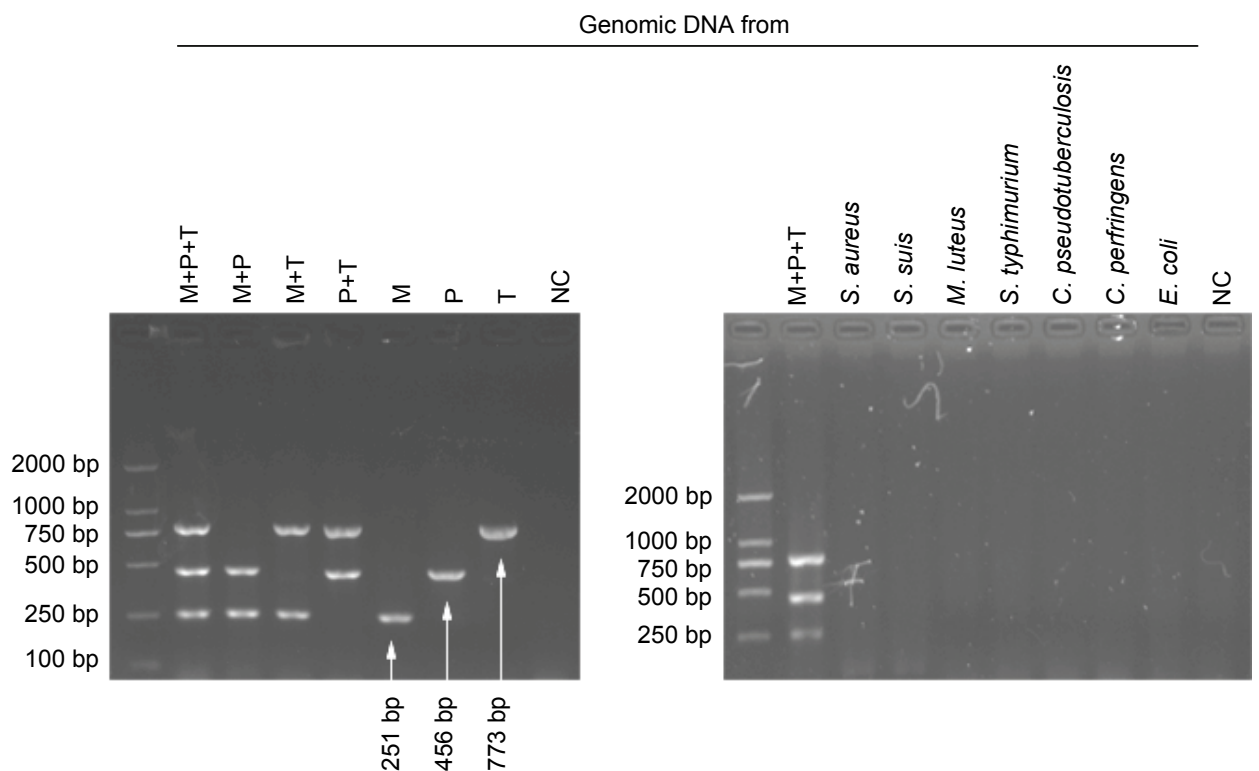

Fig. 1. Determination of the specificity of the multiplex PCR. The multiplex PCR could detect genomic DNA from Pasteurella multocida (P), Mannheimia haemolytica $(\mathrm{M})$ and Trueperella pyogenes $(\mathrm{T})$ and any combination of two or three kinds of genomic DNA from these three bacteria. In contrast, genomic DNAs from Staphylococcus aureus, Streptococcus suis, Micrococcus luteus, Salmonella typhimurium, Corynebacterium pseudotuberculosis, Clostridium perfringens and Escherichia coli could not be detected by the multiplex PCR. NC: negative control for the multiplex PCR. The white arrows indicate the amplicons representing the existence of $P$. multocida (456 bp), M. haemolytica (251 bp) and $T$. pyogenes (773 bp), respectively

\section{Determination of the sensitivity of the multiplex $P C R$}

The detection limit of the multiplex PCR was subsequently investigated by performing the reactions with a 10 -fold serial diluted genomic DNA extracted from P. multocida, M. haemolytica and T. pyogenes. As shown in Fig. 2a, the detection limit of the multiplex PCR for each kind of genomic DNA simultaneously was $40 \mathrm{pg} / \mu \mathrm{L}$. Compared with the results of conventional PCRs (Fig. 2b), which was performed under the optimised conditions, the multiplex PCR showed the same sensitivity in detecting genomic DNAs from M. haemolytica and $T$. pyogenes and a lower sensitivity in detecting genomic DNA from $P$. multocida. 

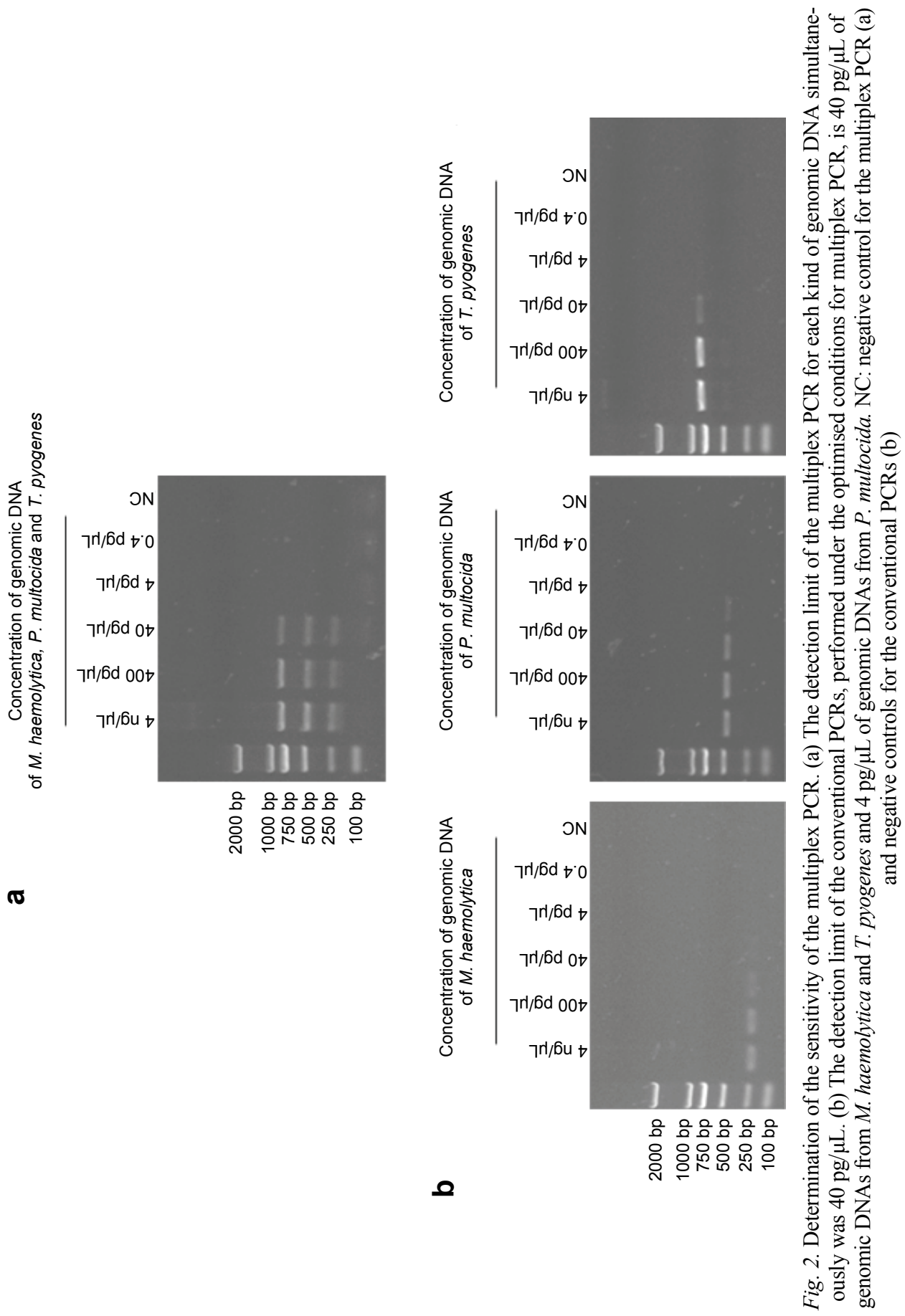

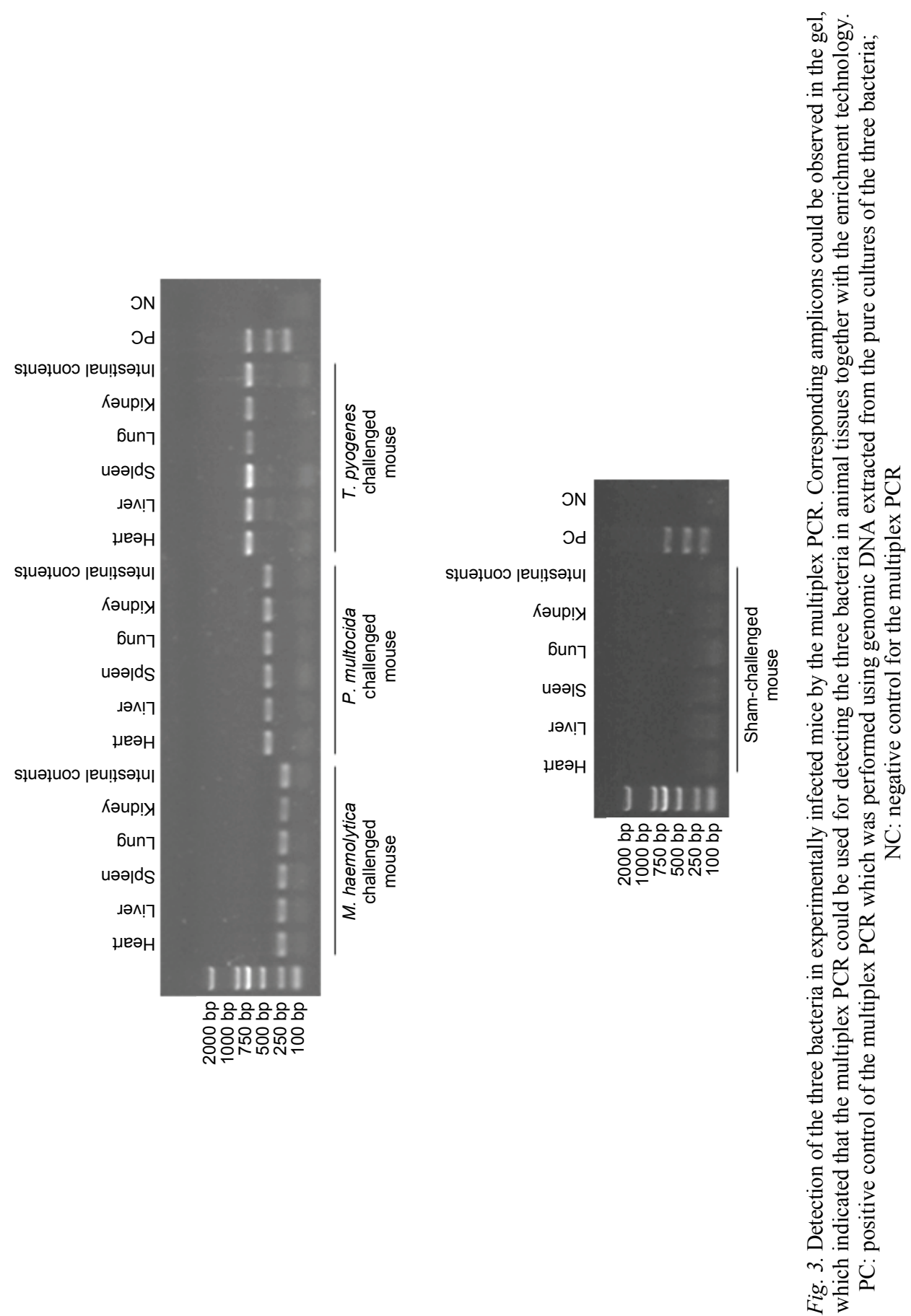
Detection of $\mathrm{P}$. multocida, M. haemolytica and T. pyogenes in experimentally infected mice by the multiplex PCR

The hearts, livers, spleens, lungs, kidneys and intestinal contents harvested from experimentally infected mice were all positive for the corresponding microorganism when detected by the multiplex PCR (Fig. 3) and by bacterial isolation (data not shown). In contrast, the specimens harvested from sham-challenged mice were all negative (Fig. 3) when detected by the multiplex PCR and by bacterial isolation.

\section{Detection of the three bacteria in artificially contaminated bovine tissues}

Figure 4 shows that detecting different artificially contaminated bovine tissues by the multiplex PCR produced results in line with our expectations.
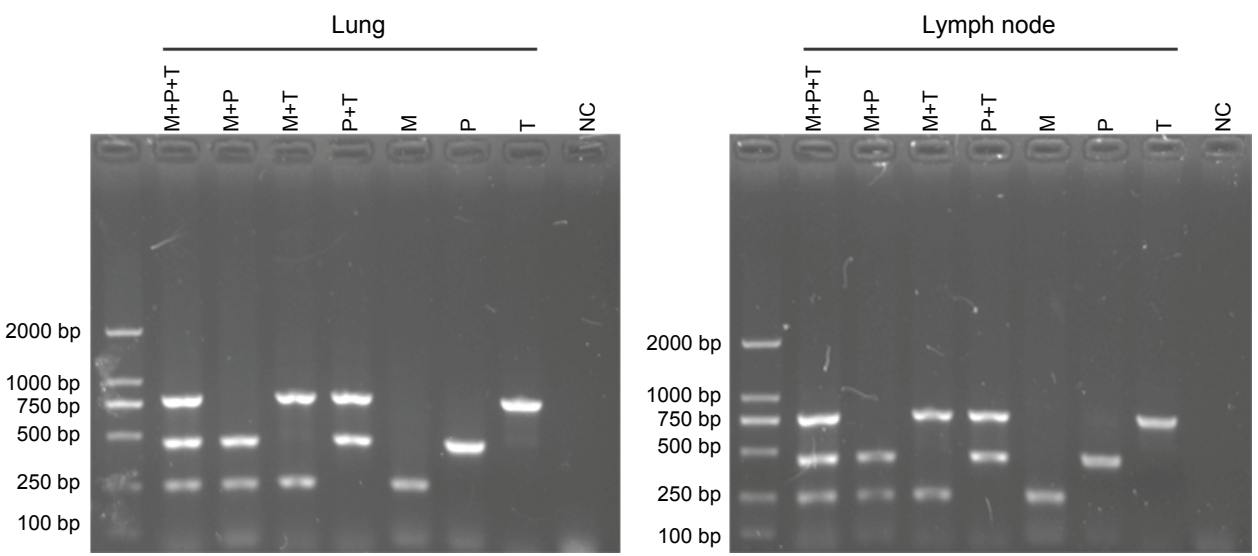

Fig. 4. Detection of the three bacteria in artificially contaminated bovine tissues. Corresponding amplicons could be observed in the gel which indicated that the multiplex PCR could be used for detecting P. multocida $(\mathrm{P})$, M. haemolytica $(\mathrm{M})$ and T. pyogenes $(\mathrm{T})$ in bovine tissues together with the enrichment technology. PC: positive control of the multiplex PCR which was performed using genomic DNA extracted from the pure cultures of the three bacteria; $\mathrm{NC}$, negative control for the multiplex PCR

\section{Discussion}

Although viral pathogens are generally the cause of primary infections during BRDC (Grissett et al., 2015), with the exception of certain instances of BRSV, fatality is usually not associated with those viral pathogens alone (Panciera and Confer, 2010). In contrast, most of the bacterial pathogens involved in BRDC usually cause a secondary infection, and the bacterial secondary infection often aggravates BRDC and leads to the animal's death (Panciera and Confer, 2010). Thus, rapid detection of these bacterial pathogens would be beneficial for 
formulating a rational plan for the treatment of BRDC. As broad-spectrum antibiotics capable of reducing the bacterial burden in the tissues are usually used for the early treatment of BRDC (Lubbers and Turnidge, 2015), the classical methods, such as bacterial isolation, are insufficiently sensitive for the detection of these bacterial pathogens, thereby hindering the formulation of prevention strategies against the spread of the disease. Thus, there is a need for establishing a rapid and sensitive detection method targeting the bacterial pathogens that play important roles in the pathogenesis of BRDC. The multiplex PCR assay is a convenient, rapid and cost-effective tool for the identification of different pathogens in a single detection (Moriconi et al., 2017). Thus, in this study we attempted to establish a multiplex PCR for the simultaneous detection of the three bacterial pathogens closely related to BRDC, namely P. multocida, M. haemolytica and $T$. pyogenes.

To obtain a good specificity, sensitivity and rapidity of the assay, factors that could affect the detection, such as the target gene as well as the concentration and the annealing temperature of the primers, must be considered. The two most critical factors are choosing the appropriate target gene and designing suitable primers. In the current study, we chose the KMT1, artJ-lktC and plo genes as target genes for the detection of P. multocida, M. haemolytica and T. pyogenes, respectively, by the multiplex PCR. These genes were reported to be highly conserved among strains of these three bacteria. In addition, some studies have enrolled these genes as a target for establishing the detection method for the three bacteria (Townsend et al., 1998; Angen et al., 2009; Aghamiri et al., 2014). Therefore, choosing these genes as the targets of the multiplex PCR would guarantee the credibility and reproducibility of the method. The primers for the multiplex PCR were designed at the discrepant region between three genes, which provided the method with the capacity of discriminating the three genes. According to our results, the primers we designed worked relatively well.

The concentration and the annealing temperature of the primers were also intensively optimised in the current study. Given that primers in the multiplex PCR reaction system will interfere with each other, the primer concentration and the annealing temperature were adjusted to obtain the highest possible efficacy for each primer pair. Based on our observations, the primer pair targeting the plo gene was more sensitive to the changes of these two parameters (data not shown). Thus, optimisation of the concentration and the annealing temperature of the PCR was mainly based on the principle of efficient acquisition of the 773-bp product through the multiplex PCR. After the optimisation of the two parameters, the multiplex PCR showed equal efficacy in obtaining all of the three products.

Other parameters, including the concentration of dNTP and $\mathrm{Mg}^{2+}$ in the reaction mixture and the annealing time and circle number of the assay, were also evaluated. However, these parameters showed less influence on the efficacy 
of the multiplex PCR compared with the parameters mentioned above, such as concentration and the annealing temperature of the primers.

The specificity of the multiplex PCR could be inferred from three aspects. The first is the perfectly matched nucleotide sequence of the amplicons with the reference sequences published in the GenBank. The second is the negative result of detection of the seven unrelated bacterial strains by the multiplex PCR. The third is the result of the specific amplification of corresponding products from bacteria proliferating from the intestinal contents of experimentally infected mice. Given that the intestinal contents of the mice contain many other different bacteria, the result suggested that the multiplex PCR could identify P. multocida, $M$. haemolytica and $T$. pyogenes specifically from a complex bacterial mixture.

The detection limit of the multiplex PCR was defined using the unit of pg genomic DNA $/ \mu \mathrm{L}$ in the total PCR reaction mixture. Although numerous studies defined the parameter using the number of colony-forming units (CFU) of the target bacterium (Hu et al., 2011; Feng et al., 2016), we thought it could not precisely define the sensitivity of our methods. The loss of some of the genomic DNA during the extraction process should be taken into account.

The multiplex PCR could precisely detect bacteria from experimentally infected mice and artificially contaminated bovine tissues, which suggested the practicability and efficiency of the method in detecting the three bacteria from clinical specimens. Although validating the method using real clinical specimens would be the best, collecting clinical specimens that are totally in accordance with our requirement, as shown in Fig. 4, is difficult. In the current study, the bacteria were first enriched in a culture medium and then detected through the multiplex PCR. Although the enrichment of the bacteria was time consuming, the total time for completing the detection was, in our experience, not longer than eight hours, which is much shorter than the time requirement of the conventional bacterial isolation method. To further reduce the time cost of the detection process, the method could be optimised for directly detecting the three bacteria in clinical specimens.

Recently, a Dembo respiratory PCR method that can simultaneously detect 16 different BRDC-related pathogens in nasal swabs from bovine animals has been established on the basis of TaqMan real-time PCR technology (Kishimoto et al., 2017). This method provides many advantages, such as identification of numerous existing pathogens and high sensitivity. The targets of this method include the three bacteria mentioned above. However, the results of this method, in our opinion, should be interpreted carefully for the following reasons. Firstly, BRDC-related bacterial pathogens are usually common commensals in the bovine upper respiratory tract. As such, the real-time PCR may yield false positive results. Thus, thresholds which can be used to discriminate the difference in bacterial pathogen burdens between healthy bovines and bovines with BRDC, must be cautiously defined. Secondly, an equal or even higher burden of Pasteurella 
and Corynebacterium in the upper respiratory tract of healthy bovines than in the upper respiratory tract of bovines suffering from pneumonia has been reported recently (Lima et al., 2016). Therefore, the findings based on detection results in samples from the bovine upper respiratory tract, may not indicate whether these bacteria play roles in pneumonia, although these results may reveal a high burden of certain bacteria in the bovine upper respiratory tract.

In contrast, the colonisation and proliferation of bacterial pathogens in bovine lungs are closely related to bovine pneumonia (Confer, 2009), and the three target bacteria can invade lymph nodes (Nattermann and Horsch, 1977; Shoo et al., 1990; Dagleish et al., 2016). Therefore, combined with the necropsy results, the detection results obtained from tissue samples could be more precise than those from bovine upper respiratory tract samples in determining whether or not these bacteria are involved in the disease.

In conclusion, we developed a multiplex PCR assay that could specifically and sensitively detect and differentiate P. multocida, M. haemolytica and T. pyogenes in cultures. This multiplex PCR, together with the bacterial enrichment technology, could also be used for detecting these three bacteria in clinical samples. The detection of the bacteria in clinical samples by multiplex PCR could be completed within eight hours. This method would be useful for the diagnosis of BRDC related to these three bacterial pathogens.

\section{Acknowledgements}

This work was supported by the National Natural Science Foundation of China (Grant No. 31402177 and No. 31672529), the China Postdoctoral Science Foundation (Grant No. 2015M571387), the Heilongjiang Postdoctoral Grant (Grant No. LBHZ14025), the 'Young Talents' Project of Northeast Agricultural University (Grant No. 16QC25) and the China Agriculture Research System (CARS-37).

\section{References}

Aghamiri, S. M., Haghkhah, M., Ahmadi, M. R. and Gheisari, H. R. (2014): Development of a multiplex PCR for the identification of major pathogenic bacteria of post-partum endometritis in dairy cows. Reprod. Domest. Anim. 49, 233-238.

Angen, O., Thomsen, J., Larsen, L. E., Larsen, J., Kokotovic, B., Heegaard, P. M. and Enemark, J. M. (2009): Respiratory disease in calves: microbiological investigations on trans-tracheally aspirated bronchoalveolar fluid and acute phase protein response. Vet. Microbiol. 137, $165-171$.

Billington, S. J., Jost, B. H., Cuevas, W. A., Bright, K. R. and Songer, J. G. (1997): The Arcanobacterium (Actinomyces) pyogenes hemolysin, pyolysin, is a novel member of the thiolactivated cytolysin family. J. Bacteriol. 179, 6100-6106.

Confer, A. W. (2009): Update on bacterial pathogenesis in BRD. Anim. Health Res. Rev. 10, 145-148. 
Dagleish, M. P., Bayne, C. W., Moon, G. G., Finlayson, J., Sales, J., Williams, J. and Hodgson, J. C. (2016): Differences in virulence between bovine-derived clinical isolates of Pasteurella multocida serotype A from the UK and the USA in a model of bovine pneumonic pasteurellosis. J. Comp. Pathol. 155, 62-71.

Elbrissi, A., Sabeil, Y. A., Khalifa, K. A., Enan, K., Khair, O. M. and El Hussein, A. M. (2017): Isolation, identification and differentiation of Campylobacter spp. using multiplex PCR assay from goats in Khartoum State, Sudan. Trop. Anim. Health Prod. 49, 575-581.

Feng, K., Hu, W., Jiang, A., Sarengaowa, Xu, Y., Zou, Y., Yang, L. and Wang, X. (2016): A dual filtration-based multiplex PCR method for simultaneous detection of viable Escherichia coli O157:H7, Listeria monocytogenes, and Staphylococcus aureus on fresh-cut cantaloupe. PloS One 11, e0166874.

Griffin, D. (2010): Bovine pasteurellosis and other bacterial infections of the respiratory tract. Vet. Clinics North Am. - Food Anim. Pract. 26, 57-71.

Grissett, G. P., White, B. J. and Larson, R. L. (2015): Structured literature review of responses of cattle to viral and bacterial pathogens causing bovine respiratory disease complex. J. Vet. Intern. Med. 29, 770-780.

Horwood, P. F. and Mahony, T. J. (2011): Multiplex real-time RT-PCR detection of three viruses associated with the bovine respiratory disease complex. J. Virol. Methods 171, 360-363.

Hu, Q., Tu, J., Han, X., Zhu, Y., Ding, C. and Yu, S. (2011): Development of multiplex PCR assay for rapid detection of Riemerella anatipestifer, Escherichia coli, and Salmonella enterica simultaneously from ducks. J. Microbiol. Methods 87, 64-69.

Kardos, G., Nagy, J., Antal, M., Bistyák, A., Tenk, M. and Kiss, I. (2007): Development of a novel PCR assay specific for Riemerella anatipestifer. Lett. Appl. Microbiol. 44, 145-148.

Kishimoto, M., Tsuchiaka, S., Rahpaya, S. S., Hasebe, A., Otsu, K., Sugimura, S., Kobayashi, S., Komatsu, N., Nagai, M., Omatsu, T., Naoi, Y., Sano, K., Okazaki-Terashima, S., Oba, M., Katayama, Y., Sato, R., Asai, T. and Mizutani, T. (2017): Development of a one-run realtime PCR detection system for pathogens associated with bovine respiratory disease complex. J. Vet. Med. Sci. 79, 517-523.

Lima, S. F., Teixeira, A. G., Higgins, C. H., Lima, F. S. and Bicalho, R. C. (2016): The upper respiratory tract microbiome and its potential role in bovine respiratory disease and otitis media. Sci. Rep. 6, 29050. doi: 10.1038/srep29050.

Lubbers, B. V. and Turnidge, J. (2015): Antimicrobial susceptibility testing for bovine respiratory disease: getting more from diagnostic results. Vet. J. 203, 149-154.

Moriconi, M., Acke, E., Petrelli, D. and Preziuso, S. (2017): Multiplex PCR-based identification of Streptococcus canis, Streptococcus zooepidemicus and Streptococcus dysgalactiae subspecies from dogs. Comp. Immunol. Microbiol. Infect. Dis. 50, 48-53.

Nattermann, H. and Horsch, F. (1977): The Corynebacterium pyogenes infection in cattle. 1. Incidence of the pathogen. Arch. Exp. Vet.-Med. 31, 405-413.

Panciera, R. J. and Confer, A. W. (2010): Pathogenesis and pathology of bovine pneumonia. Vet. Clin. North Am. - Food Anim. Pract. 26, 191-214.

Shoo, M. K., Wiseman, A., Allan, E. M., Dalgleish, R. G., Gibbs, H. A., Al-Hendi, A. B. and Selman, I. E. (1990): Distribution of Pasteurella haemolytica in the respiratory tracts of carrier calves and those subsequently infected experimentally with Dictyocaulus viviparus. Res. Vet. Sci. 48, 383-385.

Thonur, L., Maley, M., Gilray, J., Crook, T., Laming, E., Turnbull, D., Nath, M. and Willoughby, K. (2012): One-step multiplex real time RT-PCR for the detection of bovine respiratory syncytial virus, bovine herpesvirus 1 and bovine parainfluenza virus 3. BMC Vet. Res. 8, 37.

Townsend, K. M., Frost, A. J., Lee, C. W., Papadimitriou, J. M. and Dawkins, H. J. (1998): Development of PCR assays for species- and type-specific identification of Pasteurella multocida isolates. J. Clin. Microbiol. 36, 1096-1100. 